\title{
LES TERRITOIRES CRÉOLOPHONES DE L'OCÉAN INDIEN ${ }^{1}$
}

\author{
Jan Holeš
}

\begin{abstract}
The article deals with French-based creoles, with a special attention paid to those spoken on the islands in the Indian Ocean. These include the creole languages used in two independent countries, the Republic of Mauritius (and Rodrigues, its dependency) and the Republic of Seychelles, and in the French overseas department of Réunion. The article contains samples of the respective creole languages and some illustrations of their use.

Keywords: Creole; Indian Ocean; Mauritius; Rodrigues; Seychelles; Réunion.

Resumé : L'article traite des langues créoles à base française, surtout celles parlées dans les îles de l'Océan Indien. Il s'agit des créoles utilisés dans deux états indépendants : la République de Maurice (avec l'île de Rodriques, sa partie autonome) et la République des Seychelles, et dans le département d'outre-mer français de la Réunion. L'article contient les exemples des textes en créoles et quelques illustrations de leur usage.

Mots-clés : créole ; Océan Indien ; Maurice ; Rodrigues ; Seychelles ; Réunion.
\end{abstract}

\section{Introduction}

Dans cet article, nous nous proposons de passer très rapidement en revue les langues créoles à base française présentes dans le monde afin de se concentrer sur les langues créoles à base française dans l'Océan Indien, en accompagnant nos propos de quelques textes en créoles et d'illustrations de leur usage récent.

Nous avons expliqué les notions principales concernant les créoles ailleurs (Holeš 2007), constatant que les linguistes sont d'accord pour admettre que les créoles sont des langues distinctes et que leur analyse pourrait contribuer à une meilleure compréhension des problèmes généraux du langage. Dans la publication citée, nous avons expliqué la différence entre le créole et le pidgin, entre le sabir et la lingua franca, et proposé quelques étymologies du mot créole.

Même si les recherches ont beaucoup progressé, il y a toujours beaucoup de travail à effectuer dans le domaine de la description des créoles à base française. Dans le

1 L'article s'inscrit dans le cadre du projet Variété linguistique et communication (Jazyková rozmanitost a komunikace), CZ.1.07/2.3.00/20.0061, co-financé par le Fonds social européen et le budget de l'État de la République tchèque. 
contexte tchèque, quelques travaux concernant les territoires franco-créolophones ont été publiés par Jaromír Kadlec (2002a, b), parfois en collaboration avec l'auteur de cet article $(2004,2006)$.

Le lecteur trouvera une liste assez longue de langues créoles et de pidgins dans la publication de Hancock (1977), qui en répertorie 127. Pour la description des créoles en général, nous référerons aux publications de John Holm (2000) ou de Jacques Arends, Pieter Muysken et Norval Smith (1994).

Pour les créoles à base française, nous soulignons les travaux de Marie-Christine Hazaël-Massieux (par ex. 2011), Robert Chaudenson (1978, 1979, 1985, 1995), Claudine Bavoux et Didier de Robillard (2002) ainsi que Ioana Vintilă-Rădulescu (1979).

La majorité des auteurs (par ex. Hazaël-Massieux 2011 : 7) distinguent deux grandes zones géographiques où se sont développées les langues créoles à base française :

- la zone américano-caraïbe avec le louisianais (en voie de disparition), le haïtien, le guyanais et les créoles des Petites Antilles - avec les variétés de Guadeloupe, Martinique, Sainte-Lucie, Dominique et Trinidad (disparu);

- la zone de l'Océan Indien, avec le réunionnais, le mauricien (et la variété rodriguaise) et le seychellois.

On ajoute parfois encore le tayo, créole de la commune Mont-Dore en Nouvelle-Calédonie, d'une extension assez restreinte (le nombre de locuteurs estimé à deux milles, selon Gordon 2005 : 585). Dans le texte qui suit, nous nous concentrerons sur les créoles de la zone de l'Océan Indien.

\section{Zone de 1'Océan Indien}

Les créolistes s'accordent à dire que les créoles américains et ceux de l'Océan Indien sont structurellement assez similaires, beaucoup plus que par ex. les créoles à base portugaise de l'Asie et de l'Afrique (Holm 2000 : 86). Par contre, historiquement et démographiquement, leur situation est différente. Selon Valdman (1978 : 33-34), les descendants d'esclaves africains ne constituent un élément démographique important qu'aux îles Seychelles. Les îles de Maurice et de la Réunion se distinguent des Antilles créolophones par la nature multiethnique de leur population, avec une domination de descendants indiens recrutés après l'abolition de l'esclavage.

Les différentes hypothèses concernant la genèse des créoles de l'Océan Indien sont commentées par Corne (1978). Selon la majorité des auteurs, les créoles utilisés dans les îles de la Réunion, Maurice, Rodrigues et Seychelles ont évolué du "créole bourbonnais ». En effet, cette langue a été d'abord été parlée à la Réunion qui portait le nom d'Île Bourbon jusqu'en 1793 et qui a été peuplée durablement par les colons français dès 1665. Elle s'est ensuite propagée sur l'île Maurice et, de là, sur l'île Rodrigues et les Seychelles. Le créole s'est différencié à cause de l'insularité et donc de l'isolation des territoires et les différences entre ces créoles ont été accentuées par l'évolution historique diverse et par les vagues d'immigration d'origines différentes (par ex. la colonisation anglaise de l'île Maurice).

\subsection{Maurice et Rodrigues}

L'île Maurice, état indépendant membre du Commonwealth, est située au sud-est de l'Afrique et à environ $870 \mathrm{~km}$ à l'est de Madagascar. Le territoire se compose de l'île 
principale, où se trouve aussi la capitale (Port-Louis), de Rodrigues (l'île volcanique, à $560 \mathrm{~km}$ à l'est de Maurice) et de l'archipel d'Agaléga (situé à $1070 \mathrm{~km}$ au nord) et des écueils de Cargados Carajos (à $390 \mathrm{~km}$ au nord-nord-est). La République de Maurice compte à peu près 1,3 millions d'habitants. La population de l'île de Rodrigues est de 35 mille habitants.

Jacques Leclerc (2012) résume les résultats du recensement démographique de 2000 qui révélait que les langues parlées quotidiennement sont le créole, par 826152 locuteurs $(70,0 \%)$, le bhojpouri (langue parlée surtout dans le nord-est de l'Inde par quelque 150 millions de locuteurs) par 142387 locuteurs $(12,0 \%)$ et le français, par 39953 locuteurs $(3,3 \%)$. Il est relativement difficile de déterminer le nombre de locuteurs des langues présentes sur les îles parce que la majorité de la population parle plusieurs langues. D'autres statistiques établissent plutôt à $80,5 \%$ le pourcentage du créole, à $12,1 \%$

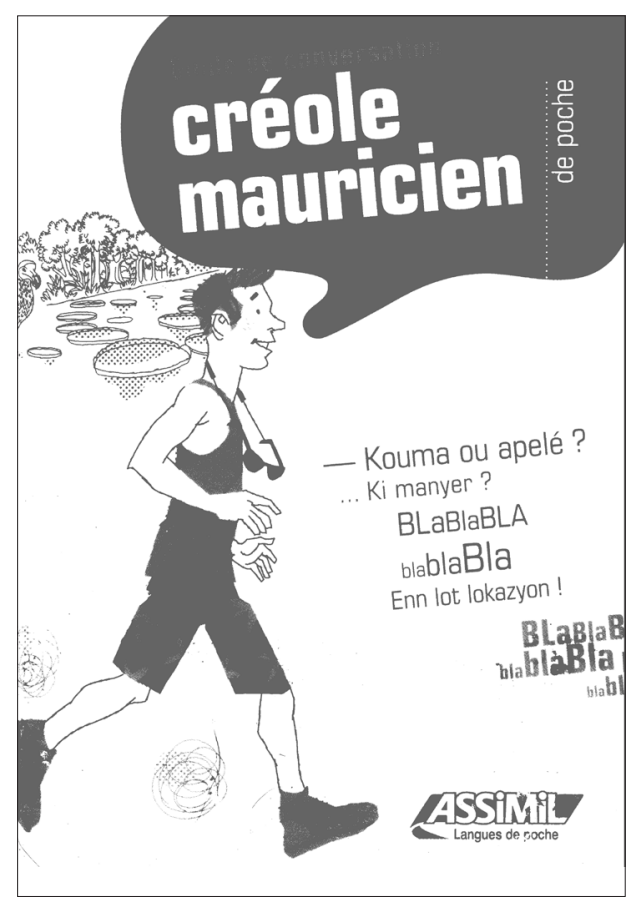
celui du bhojpouri, à 3,4\% celui du français Manuel de créole mauricien publié par Assimil et à moins de $1 \%$ celui de l'anglais. Il resterait (Paris 2011).

ainsi 3,7 \% pour toutes les autres langues. Le nombre d'usagers du bhodjpouri et des autres langues indiennes diminue et les locuteurs créolophones sont de plus en plus nombreux. La Constitution ne se prononce pas sur le statut officiel des langues. Juridiquement, la République n'a pas défini sa langue officielle, mais dans les faits, c'est l'anglais qui joue le rôle de la langue officielle de la République. Le créole, langue dominante, est utilisé sur la totalité de l'île (Leclerc 2012).

La langue maternelle de 37 mille habitants est le français, l'anglais étant la langue maternelle de trois mille habitants. Pour le reste de la population, l'anglais et le français sont des langues étrangères. La variante du créole local, légèrement différente du créole mauricien, est la langue maternelle et la langue la plus couramment utilisée pour $96 \%$ des habitants de Rodrigues, territoire ethniquement et linguistiquement assez homogène.

En tant qu'exemple du créole mauricien (aussi appelé mauricien ou morisien/morisyen en créole) nous nous servons d'un passage (adapté) du Petit Prince, traduit par Dev Virahsawmy, linguiste, poète, dramaturge, traducteur et surtout défenseur du créole mauricien. Nous ajoutons la traduction en créole réunionnais dont nous traiterons plus bas (traduction de Jocelin Lakia, Lo Pti Prins, Caraïbeditions, 2010) ainsi que la version originale française. 


\begin{tabular}{l} 
Créole mauricien \\
Kan mo ti ena si-zan, enn zour mo ti trouv enn zoli-zoli zimaz dan enn liv. Liv la ti lor Lafore Vierz e li ti apel \\
Bann Zistoir Vre. Zimaz la ti montre enn serpan boa ki ti pe aval enn zanimo feros. \\
Dan liv la ti dir ki bann boa zot aval zot manze antie, san kraze, san mase. Lerla zot nepli kapav bouze. Zot dormi \\
pandan sis moi pou dizer zot manze. \\
Sa zistoir la ti bien travay mo latet e li ti donn moi lide pou mo fer mo premie desin. Mo montre mo desin bann \\
gran dimoun; mo dimann zot si zot pa gagn per. \\
Zot dir moi sapo pa fer dimoun per. \\
Créole réunionnais \\
Kan moin lavé sizan moin la vi, in foi, in gayar zimaz dann in liv dsi la Foré Vièrz, sa té i apèl Zistoir Vré. Dési \\
lavé in serpan boa té i anval in zanimo sovaz. \\
Dann liv la, lété ékri : "bann serpan boa i anval zot proi an antié, san mashé. Apré sa, zot i guingn pi bouzé, zot \\
i dor pandan si moi pou dizéré. » \\
Alors, moin la réfléshi in ta dsi sak i ariv dann la zing. A mon tour, moin la guingn tras mon promié désin. Moin \\
la montr mon shédèv bann gran moun, moin la dmann a zot si mon désin té i fé pèr a zot. \\
Zot la réponn a moin: " par koman in shapo i pé fé pèr? ? \\
\hline Français \\
\hline Lorsque j'avais six ans j'ai vu, une fois, une magnifique image, dans un livre sur la Forêt Vierge qui s'appelait « \\
Histoires Vécues ". Ça représentait un serpent boa qui avalait un fauve. \\
On disait dans le livre: "Les serpents boas avalent leur proie tout entière, sans la mâcher. Ensuite ils ne peuvent \\
plus bouger et ils dorment pendant les six mois de leur digestion ». \\
J'ai alors beaucoup réfléchi sur les aventures de la jungle et, à mon tour, j'ai réussi, avec un crayon de couleur, \\
à tracer mon premier dessin. J'ai montré mon chef d'œuvre aux grandes personnes et je leur ai demandé si mon \\
dessin leur faisait peur. \\
Elles m'ont répondu: « Pourquoi un chapeau ferait-il peur ? » \\
\hline
\end{tabular}

\subsection{Réunion}

La Réunion, à la fois département et région d'outre-mer appartenant à la France, est située à 1800 km à l'est de Madagascar et à 170 km au sud-ouest de l'île Maurice. Le territoire comptait, en 2011, 840 mille habitants.

Le créole réunionnais a été décrit en détail par Beniamino (1996), la situation linguistique de l'île par Kadlec et Holeš (2002a). Valdman (1978 : 34) souligne l'importance de l'ancienne île de Bourbon pour la diffusion du créole dans l'Océan Indien (le créole bourbonnais, voir plus haut). À la Réunion, on distingue trois variétés du créole: a) le créole des Bas, utilisé par les réunionnais d'origine africaine, malgache et indienne, b) le créole des Hauts, pratiqué par les Blancs des Hauts et c) le créole urbain, fortement francisé.

La société réunionnaise est originale par la diversité de ses origines, due à une immigration permanente depuis plus de trois siècles. Selon Leclerc (2012), on trouve parmi les habitants $35 \%$ de Réunionnais d'origine africaine - les cafres, fortement métissés. La population blanche, d'origine européenne, représenterait $25 \%$ de l'ensemble auquel il faut ajouter environ $5 \%$ de Métropolitains (les Zoreils) résidant sur l'île. Suivent environ $25 \%$ d'Indiens tamouls (Malabars) constitués des descendants des travailleurs arrivés, de 1848 à 1882, de la côte orientale de l'Inde et de la côte de Malabar. Chaudenson (1978) note qu'entre le créole réunionnais et le français existe un continuum linguistique où intervient également un français créolisé parlé essentiellement par le groupe dit des «Petits Blancs ». La langue maternelle de la majorité de la population (plus de 55 \%) est le créole réunionnais, utilisé surtout sous la forme parlée et dans les conversations informelles. 
Selon Gordon (2005 : 178), on compterait 2400 locuteurs ayant le français comme langue maternelle, 554 mille locuteurs créolophones (donnée de 1987) et 120 mille usagers de tamoule (y compris les personnes qui le parlent comme langue seconde).
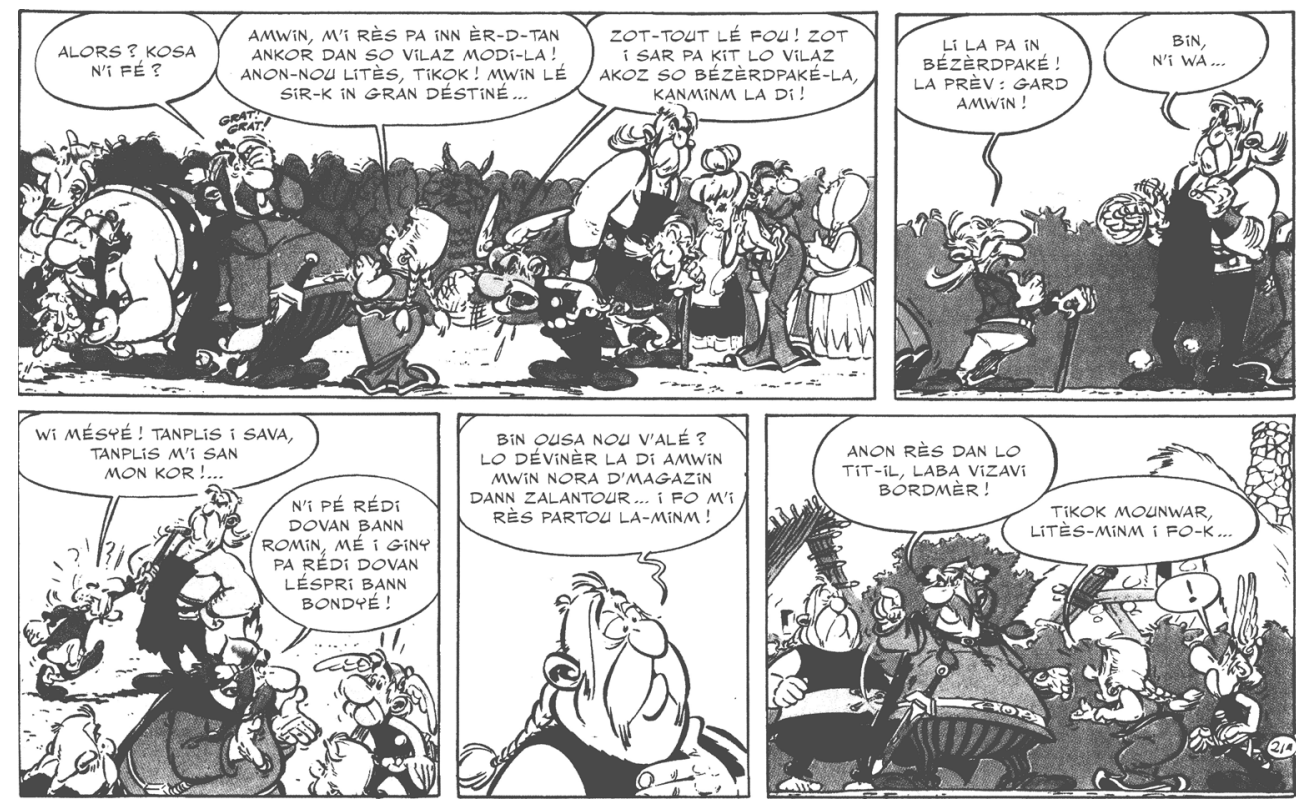

Extrait de la traduction d'un album d'Astérix en créole réunionnais. Goscinny, René - Uderzo, Albert (2009) : Lo Dévinèr, Paris : Caraïbeditions.

\subsection{Seychelles}

La République des Seychelles se compose de 115 îlots situés au nord-est de Madagascar, à peu près à $1800 \mathrm{~km}$ des côtes africaines, avec l'île principale de Mahé, centre de la vie politique et économique, qui abrite la capitale, Victoria. Quelque quarante îles peuplées ont une population de 86 mille habitants et la République des Seychelles est donc le pays ayant la population la moins élevée de toute l'Afrique.

Le créole seychellois a été relativement bien décrit grâce aux œuvres de Bollée (1977, 1993), Corne (1977) et D'Offay et Lionnet (1982). La majorité de la population est d'origine africaine ce qui différencie les Seychelles de l'île Maurice et de la Réunion. Outres les noirs, on y retrouve les Indiens, les Chinois et les Européens. Quelque 95 \% des habitants utilisent le créole seychellois qui est la langue de la vie quotidienne et de la culture populaire. Le seychellois est également l'une des trois langues officielles de la République, avec l'anglais et le français. Avec Haïti, la République des Seychelles est l'unique pays du monde ayant déclaré un créole à base française comme langue officielle. Dès les années quatre-vingt du siècle dernier, cette langue est couramment appelée le seselwa, nom proposé par le Lenstiti Kreol dans le but de promouvoir la langue maternelle de la population.

Le Lenstiti Kreol a été fondé en 1981 dans le but de faciliter la promotion du créole en tant que langue officiel, dans ses formes parlée et écrite, en tant que langue d'enseignement et langue de la littérature seychelloise. Le rôle principal du Lenstiti Kreol est de di- 


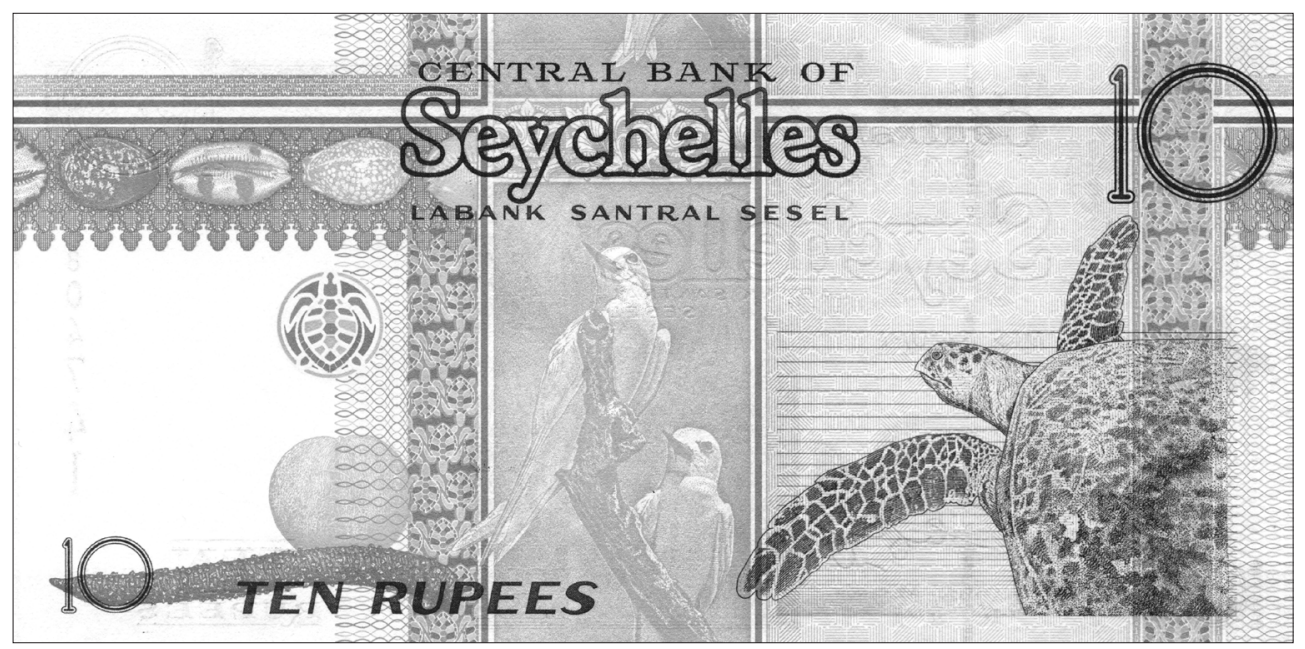

Sur les billets de banque seychellois figurent le seselwa et l'anglais.

riger le développement linguistique et culturel de la langue pour qu'elle puisse remplir ces fonctions. Les trois missions de l'institut sont donc: a) développer la langue créole, b) développer la littérature créole et c) promouvoir l'Institut et ses services localement et à l'étranger (Lenstiti kreol 2012).

La transition du bilinguisme au trilinguisme officiel est décrite par Marie-Thérèse Choppy et Laureena Barbe (1989). Avant 1979, le créole n'avait pas de statut légal et on a répété qu'il n'était pas capable de véhiculer des idées avancées en sciences et en technologie. Dans les années 1978-81, le gouvernement a adopté une politique de bilinguisme avec pour objectif de rendre chaque Seychellois bilingue français-anglais, en excluant le créole. En 1981, le gouvernement a pris la décision de donner au seselwa un rôle primordial dans la nouvelle politique éducative et il est devenu première langue officielle, suivi de l'anglais et du français.

Le créole local n'est pas du tout homogène. Selon Leclerc (2012), on distingue le créole fin, créole fortement francisé et parlé par la bourgeoisie, le gros créole, utilisé par les classes populaires, le créole grand-bois que l'on retrouve dans les campagnes, le gros créole mozambique des paysans d'origine africaine et le créole des bulletins d'information de la radio-télévision ou de certains discours de personnalités politiques, qui semble un peu artificiel et qui contient des emprunts à l'anglais. Le créole seychellois demeure relativement proche du créole mauricien mais assez éloigné du créole réunionnais. La plupart des habitants parlent l'anglais et le français comme langues secondes. Le français est la langue des grandes événements de la vie comme les demandes en mariage, les chansons dites « romantiques », les avis de décès, etc., mais aussi des médias écrits, la lecture en général et l'affichage commercial. L'anglais reste la langue de l'Administration, du commerce, des affaires internationales et souvent de la télévision. Chaudenson (1978:10) note que l'anglais n'est la langue maternelle que de $3 \%$ des habitants et le français de $2 \%$.

Pour donner un exemple, nous avons choisi le texte de l'hymne national de la République, accompagné de sa traduction française. 
Sesel ou menm nou sel patri.

Kot nou viv dan larmoni.

Lazwa, lanmour ek lape.

Nou remersye Bondye.

Preserv labote nou pei.

Larises nou losean.

En leritaz byen presye.

Pour boner nou zanfan.

Reste touzour dan linite.

Fer monte nou paviyon.

Ansanm pou tou leternite.

Koste Seselwa!
Seychelles, notre seule patrie

Où nous vivons en harmonie

La joie, l'amour et la paix

Nous remercions le Bon Dieu!

Préservons la beauté de notre pays

La richesse de notre océan

Un héritage très précieux

Pour le bonheur de nos enfants

Restons toujours unis

Élevons notre drapeau

Ensemble pour l'éternité

Unissons-nous Seychellois!

\section{Conclusion}

Dans l'Océan Indien on retrouve les créoles à base française dans deux états indépendants : la République de Maurice (avec l'île de Rodrigues, sa partie autonome) et la République des Seychelles, ainsi que dans la région et le département d'outre-mer française de la Réunion. Seul le créole seychellois a gagné le statut officiel, même si les trois créoles (avec leurs variétés locales) sont parlés comme langues maternelles par la majorité de la population de toutes les îles. Notre article contient quelques données démolinguistiques concernant les langues répandues sur les trois territoires, les échantillons des textes dans les trois créoles et quelques illustrations de leur usage.

\section{Bibliographie}

Arends, Jacques - Muysken, Pieter - Smith, Norval (1994) : Pidgins and Creoles, Amsterdam/Philadelphia : John Benjamins Publishing Company.

Bavoux, Claudine - Robillard, Didier de (2002) : Linguistique et créolistique, Paris : Anthropos.

Beniamino, Michel (1996) : Le français de la Réunion. Inventaire des particularités lexicales, Vanves : EDICEF.

Bollée, Annegret (1977) : Le Créole Français des Seychelles, Tübingen : Max Niemeyer Verlag.

Bollée, Annegret (dir.) (1993) : Dictionnaire étymologique des créoles français de l'Océan Indien, Hamburg : Helmut Buske Verlag.

CHOpy, Marie-Thérèse - BARBE, Laureena (1989): « Seychelles : tout en seselwa », in : Diagonales 12. Octobre, 24-26.

CoRne, Chris (1977) : Seychelles Creole Grammar, Tübingen : TBL Verlag Gunter Narr.

D'Offay, Danielle - Lionnet, Guy (1982) : Diksyonner kreol-franse. Dictionnaire créole seychellois-français, Hamburg: Helmut Buske Verlag.

CHAudenson, Robert (éd.) (1978) : Les parlers créoles, Langue française 37.

CHAUdENSON, Robert (1979) : Les créoles français, Paris : Nathan.

Chaudenson, Robert (1985) : "Les Créoles », in : Antoine, Gérard - Martin, Robert (éds.) : Histoire de la langue française 1880-1914, Paris : CNRS, 415-420.

Chaudenson, Robert (1995) : " Les Créoles », in : Antoine, Gérard - Martin, Robert (éds.) : Histoire de la langue française 1914-1945, Paris : CNRS, 861-870.

CORNe, Chris (1978) : " Proto-créole et liens génétiques dans l'Océan Indien », in : CHAUDENSON, Robert (éd.) : Les parlers créoles, Langue française 37, 60-75.

Gordon, Raymond G. (éd.) (2005) : Ethnologue. Languages of the World, $15^{\text {th }}$ edition, 
Dallas : SIL International.

HANCOCK, Ian (1977) : « Repertory of Pidgin and Creole Languages », in : Pidgin and Creole Linguistics, 362-391.

HAZAËL-MASSIEUX, Marie-Christine (2011) : Les créoles à base française, Paris : OPHRYS.

Holeš, Jan (2007) : "Créoles à base française ", in : Acta Universitatis Palackianae, Philologica, Romanica Olomucensia XIX, Olomouc : Univerzita Palackého, 25-33.

HolEš, Jan - KADLEC, Jaromír (2012) : Jazyková politika frankofonních zemí, Olomouc : Univerzita Palackého v Olomouci.

Holm, John (2000) : An Introduction to Pidgins and Creoles, Cambridge : Cambridge University Press.

KADLEC, Jaromír - Holeš, Jan (2002a) : « Jazyková situace a podoba francouzštiny na ostrově Réunion », Slovo a Slovesnost 63, 287-298.

KADLEC, Jaromír (2002b) : « Postavení a podoba francouzštiny na Haiti na konci 20. století », Časopis pro moderní filologii 84, 38-49.

KADLEC, Jaromír - HolEš, Jan (2004) : « Jazyk na periferii (jazyková situace a podoba francouzštiny ve Francouzské Guayaně) », in : PoŘízKA, Petr - POLÁcH, Vladimír P. (éds.) : Vztah langue a parole v perspektivě interaktivního obratu v lingvistickém zkoumání, Olomouc: Univerzita Palackého, 141-149.

KADLEC, Jaromír - Holeš, Jan (2006) : Francouzština na americkém kontinentě, Olomouc : Univerzita Palackého.

LECLERC, Jacques (2007) : L'aménagement linguistique dans le monde, http:/ / www.tlfq. ulaval.ca/axl [en ligne le 29/09/2012].

Lensiti KreOL (2012), http:/ / www.creoleinstitute.sc [en ligne le 29/09/2012].

VALDMAN, Albert (1978) : Le créole: structure, statut et origine, Paris : Klincksieck.

VinTILĂ-RăDulescu, Ioana (1979) : Le Créole Français, The Hague/Paris/New York : Mouton.

\author{
Jan Holeš \\ Katedra romanistiky \\ Filozofická fakulta \\ Univerzita Palackého v Olomouci \\ Křížkovského 10 \\ 77180 Olomouc \\ République tchèque \\ jan.holes@upol.cz
}

